\title{
THE PHOSPHATASE CONTENT OF THE BLOOD SERUM IN JAUNDICE ${ }^{1}$
}

\author{
By CARL H. GREENE, HOWARD F. SHATTUCK AND \\ LILLIAN KAPLOWITZ
}

(From the Department of Medicine and the Laboratory of Biochemistry, New York Post-Graduate Medical School and Hospital, New York City)

(Received for publication July 16,1934)

Enzymes which hydrolyze esters of phosphoric acid, phosphatases, are found in many mammalian tissues. Robison (9) has stressed the presence of these enzymes in growing bone and their importance in relation to the processes of calcification and bone development. Further evidence of the importance of this enzyme in the metabolism of bone (6) was adduced with the discovery that the phosphatase content of the blood serum was increased in various diseases such as rickets, Paget's disease or hyperparathyroidism in which osseous changes are marked. In these conditions the increase in the serum or plasma phosphatase apparently is secondary to the osseous lesions.

Phosphatases are present in many tissues and occur in greatest amount in the intestinal mucosa and the kidney. Smaller amounts have been reported in the liver and in the bile (4). Bodansky and Jaffe $(2,3)$ have found that in normal individuals the phosphatase content of the serum varies with digestive activity and so postulate that while some of the phosphatase in the serum may be osseous in origin some at least is nonosseous. This is also true in some pathological conditions for Roberts $(7,8)$ who was one of the first to study the changes in the phosphatase content of the serum in disease, reported that high values were present in cases of obstructive jaundice whereas in catarrhal jaundice the value was only slightly increased over the normal. Roberts (8) considered this difference to be sufficiently marked to be of diagnostic value in the differentiation of the several types of jaundice. Bodansky and Jaffe (2) confirmed the increase in the serum phosphatase in jaundice but their results cast doubt on the diagnostic value of the test for they reported a series of nine cases of catarrhal jaundice and hepatitis in which elevated readings were obtained.

\section{METHODS AND MATERIALS}

Using the method of Bodansky (1) we have studied the phosphatase content of the serum in a series of 40 cases of jaundice of various types.

${ }^{1}$ Aided by a grant from the Josiah Macy Jr. Foundation. 
Determinations in a series of 73 miscellaneous cases, representative of the general run of hospital patients, and a series of 33 cases of chronic cholecystitis or cholelithiasis without jaundice were used as controls. The phosphatase content of the bile obtained by duodenal drainage was also determined in a series of 15 cases.

\section{RESULTS IN CONTROL CASES}

The determination of enzymatic activity is difficult and the results vary with the details of the particular method used so that of ten the data of different investigators cannot be compared directly. This is particularly true in the study of the phosphatase content of the serum. Roberts (8) considers 5.5 units as the upper limit of normal but in cases of jaundice does not think the results of clinical significance unless values of 10 units or over are obtained. Bodansky and Jaffe (3) consider the normal range for adults to vary from 1.5 to 4.0 units, while in children it is 5.0 to 13.0 units. Under the conditions of routine hospital use we have found much greater variations than these. The mean of the control cases was 6.3 units with a standard deviation of 2.2, while the mean of the series of cases of chronic cholecystitis without jaundice was 7.3 with a standard deviation of 2.9 units. The increase of the mean value for the serum phosphatase in the cases of cholecystitis when compared with the control series is suggestive but when subjected to statistical analysis is not sufficiently great to be conclusive. Under these conditions values between 2.0 and 11.0 may be considered as without definite pathological significance.

\section{RESULTS IN CASES OF JAUNDICE}

The results obtained in the different types of jaundice are shown in the accompanying tables. Table I comprises 8 cases of obstructive jaundice due to extrinsic pressure on the common duct, usually from carcinoma of the head of the pancreas. Jaundice was present in all, and the icterus index and serum bilirubin were elevated. The phosphatase was elevated in the majority of cases. Especial interest attaches to Case 8 in which the biliary obstruction was due to pressure from an echinoccus cyst for readings were obtained both before the onset of obstruction and after its surgical relief.

Ten cases of jaundice due to chronic cholecystitis with cholelithiasis or choledocholithiasis are given in Table II. The serum phosphatase was elevated in all though to a variable degree, the highest reading, 64.5 units, being obtained in Case 13 in which there was very little jaundice but in which there was a marked cholangitis. Cases $9,11,13$, and 17 all show a reduction in the phosphatase reading following operation and relief of the obstruction but the changes in the serum phosphatase are not as marked as those in the serum bilirubin. There was a series of colics in Case 10 
TABLE I

The phosphatase content of the serum in obstructive jaundice

\begin{tabular}{|c|c|c|c|c|c|c|c|c|c|}
\hline 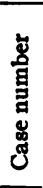 & 选 & 苟 & $\mathrm{Da}$ & ate & 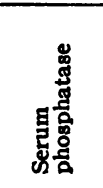 & 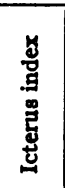 & 是崫 & 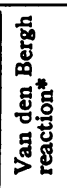 & Diagnosis \\
\hline & years & & & & $\begin{array}{l}\text { units per } \\
100 \mathrm{cc.}\end{array}$ & & $\begin{array}{l}\operatorname{mgm} . \\
\text { per. } \\
100 \mathrm{cc} .\end{array}$ & & \\
\hline 1 & 66 & M. & $\begin{array}{l}\text { March } \\
\text { March }\end{array}$ & $\begin{array}{r}2,1933 \\
20,1933\end{array}$ & $\begin{array}{l}63.2 \\
55.9\end{array}$ & $\begin{array}{l}200 \\
200\end{array}$ & $\begin{array}{l}23.5 \\
18.8\end{array}$ & $\begin{array}{l}+ \\
+\end{array}$ & $\begin{array}{l}\text { Carcinoma pancreas? } \\
\text { Clinical diagnosis }\end{array}$ \\
\hline 2 & 57 & M. & Septembe & r 30, 1933 & 22.6 & 41 & 6.0 & + & $\begin{array}{l}\text { Carcinoma stomach and } \\
\text { pancreas. }\end{array}$ \\
\hline & & & October & 4,1933 & 14.5 & 45 & 6.2 & + & $\begin{array}{l}\text { Operation September 29, } \\
1933\end{array}$ \\
\hline 3 & 32 & M. & December & 19,1932 & 20.9 & 150 & 18.7 & + & Carcinoma pancreas \\
\hline 4 & 61 & M. & $\begin{array}{l}\text { February } \\
\text { February }\end{array}$ & $\begin{array}{r}9,1933 \\
14,1933\end{array}$ & $\begin{array}{l}2.3 \\
6.4\end{array}$ & $\begin{array}{l}100 \\
111\end{array}$ & $\begin{array}{l}14.0 \\
13.6\end{array}$ & $\stackrel{+}{+}$ & $\begin{array}{l}\text { Carcinoma pancreas } \\
\text { Hepatic metastasis }\end{array}$ \\
\hline 5 & 63 & M. & $\begin{array}{l}\text { February } \\
\text { February }\end{array}$ & $\begin{array}{l}13,1934 \\
23,1934\end{array}$ & $\begin{array}{l}29.5 \\
29.5\end{array}$ & $\begin{array}{l}94 \\
75\end{array}$ & $\begin{array}{r}10.6 \\
9.5\end{array}$ & $\begin{array}{l}+ \\
+\end{array}$ & Carcinoma of liver \\
\hline 6 & 65 & M. & September & 26,1933 & 36.4 & 12 & 2.9 & + & $\begin{array}{l}\text { Carcinoma gallbladder; } \\
\text { metastasis to liver; as- } \\
\text { cites }\end{array}$ \\
\hline 7 & 44 & M. & $\begin{array}{l}\text { October } \\
\text { October } \\
\text { October }\end{array}$ & $\begin{array}{l}14,1933 \\
23,1933 \\
27,1933\end{array}$ & $\begin{array}{l}11.0 \\
11.2 \\
\\
10.5\end{array}$ & $\begin{array}{l}94 \\
39 \\
33\end{array}$ & $\begin{array}{l}7.3 \\
3.2 \\
2.9\end{array}$ & $\begin{array}{l}+ \\
+ \\
+\end{array}$ & $\begin{array}{l}\text { Pancreatitis or carcinoma } \\
\text { Operation October 18, } \\
1933\end{array}$ \\
\hline 8 & 56 & M. & $\begin{array}{l}\text { July } \\
\text { January } \\
\text { January } \\
\text { January }\end{array}$ & $\begin{array}{r}22,1933 \\
5,1934 \\
16,1934 \\
25,1934\end{array}$ & $\begin{array}{l}14.2 \\
34.6 \\
24.5 \\
11.4\end{array}$ & $\begin{array}{r}9 \\
83 \\
53 \\
19\end{array}$ & $\begin{array}{c}\text { Trace } \\
12.9 \\
7.2 \\
2.6\end{array}$ & $\begin{array}{l}0 \\
+ \\
+ \\
+\end{array}$ & $\begin{array}{l}\text { Echinococcus cysts of liver } \\
\text { Operation January } 6,1934\end{array}$ \\
\hline
\end{tabular}

* + Direct Van den Bergh reaction.

0 Indirect Van den Bergh reaction.

with intermittent obstruction and consequent marked fluctuation in the serum bilirubin level. The phosphatase showed similar fluctuations but the changes were much less striking than those in the bilirubin.

Twelve cases of hepatitis of one type or another were studied (Table III). Seven of these were of the type ordinarily referred to as catarrhal jaundice. Two were cases of syphilitic hepatitis and there was one case of toxic hepatitis following arsphenamine. The phosphatase was elevated in eight of these cases. The highest reading was obtained in Case 23 in which the serum bilirubin was most markedly increased. In general, how- 
TABLE II

The phosphatase content of the serum in obstructive jaundice

\begin{tabular}{|c|c|c|c|c|c|c|c|c|c|}
\hline 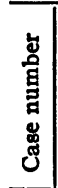 & 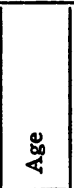 & 范 & Dat & & 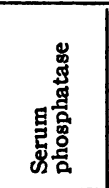 & 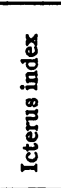 & 蝜 & 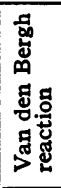 & Diagnosis \\
\hline & years & & & & $\begin{array}{l}\text { units per } \\
100 \mathrm{cc} .\end{array}$ & & $\begin{array}{c}\text { mgm. } \\
\text { per } \\
100 \mathrm{cc} .\end{array}$ & & \\
\hline 9 & 62 & F. & $\begin{array}{l}\text { November } \\
\text { November } \\
\text { January } \\
\text { January } \\
\text { February } \\
\text { March }\end{array}$ & $\begin{array}{r}22,1933 \\
29,1933 \\
17,1934 \\
31,1934 \\
28,1934 \\
7,1934\end{array}$ & $\begin{array}{l}57.7 \\
46.4 \\
39.2 \\
36.1 \\
47.9 \\
53.0\end{array}$ & $\begin{array}{r}44 \\
47 \\
23 \\
33 \\
136 \\
120\end{array}$ & $\begin{array}{r}7.2 \\
6.0 \\
2.3 \\
2.2 \\
17.9 \\
16.0\end{array}$ & $\begin{array}{l}+ \\
+ \\
+ \\
+ \\
+ \\
+\end{array}$ & $\begin{array}{l}\text { Common duct stone } \\
\text { Obstructive cirrhosis }\end{array}$ \\
\hline 10 & 65 & M. & $\begin{array}{l}\text { March } \\
\text { April }\end{array}$ & $\begin{array}{r}18,1933 \\
7,1933\end{array}$ & $\begin{array}{l}64.5 \\
35.3\end{array}$ & $\begin{array}{l}37 \\
15\end{array}$ & $\begin{array}{l}2.8 \\
1.0\end{array}$ & $\begin{array}{l}+ \\
+\end{array}$ & $\begin{array}{l}\text { Chronic cholecystitis with } \\
\text { stones } \\
\text { Cholangitis. Cholecyst- } \\
\text { ectomy March 12, } 1933\end{array}$ \\
\hline 11 & 25 & M. & $\begin{array}{l}\text { February } \\
\text { February }\end{array}$ & $\begin{array}{l}14,1933 \\
16,1933\end{array}$ & $\begin{array}{r}42.0 \\
26.4\end{array}$ & 23 & $\begin{array}{l}2.8 \\
\text { Less } \\
\text { than 2 }\end{array}$ & $\begin{array}{l}+ \\
0\end{array}$ & Common duct stone \\
\hline 12 & 60 & F. & $\begin{array}{l}\text { April } \\
\text { April } \\
\text { May } \\
\text { May }\end{array}$ & $\begin{array}{l}12,1933 \\
24,1933 \\
22,1933 \\
23,1933\end{array}$ & $\begin{array}{l}31.6 \\
19.3 \\
20.8 \\
18.6\end{array}$ & $\begin{array}{r}120 \\
75 \\
250 \\
40\end{array}$ & $\begin{array}{r}19.5 \\
4.0 \\
15.8 \\
9.0\end{array}$ & $\begin{array}{l}+ \\
+ \\
+\end{array}$ & $\begin{array}{l}\text { Chronic cholecystitis with } \\
\text { stones } \\
\text { Following colic } \\
\text { After duodenal drain- } \\
\text { age }\end{array}$ \\
\hline 13 & 48 & M. & $\begin{array}{l}\text { November } \\
\text { Novémber }\end{array}$ & $\begin{array}{r}5,1933 \\
10,1933\end{array}$ & $\begin{array}{l}21.7 \\
14.0\end{array}$ & $\begin{array}{r}125 \\
71\end{array}$ & $\begin{array}{r}15.7 \\
5.5\end{array}$ & $\begin{array}{l}+ \\
+\end{array}$ & $\begin{array}{l}\text { Chronic cholecystitis with } \\
\text { stones } \\
\text { Cholecystectomy No- } \\
\text { vember } 3,1933\end{array}$ \\
\hline 14 & 70 & F. & $\begin{array}{l}\text { October } \\
\text { November }\end{array}$ & $\begin{array}{r}10,1933 \\
2,1933\end{array}$ & $\begin{array}{l}17.5 \\
11.6\end{array}$ & $\begin{array}{r}100 \\
21\end{array}$ & $\begin{array}{r}11.3 \\
2.0\end{array}$ & $\begin{array}{l}+ \\
+\end{array}$ & $\begin{array}{l}\text { Chronic cholecystitis with } \\
\text { stones } \\
\text { Cholecystectomy October } \\
9,1933\end{array}$ \\
\hline 15 & 46 & M. & $\begin{array}{l}\text { October } \\
\text { October } \\
\text { November }\end{array}$ & $\begin{array}{l}17,1933 \\
23,1933 \\
20,1933\end{array}$ & $\begin{array}{l}12.6 \\
14.0 \\
13.4\end{array}$ & $\begin{array}{r}108 \\
65\end{array}$ & $\begin{array}{r}10.7 \\
6.0 \\
2.0\end{array}$ & $\begin{array}{l}+ \\
+ \\
+\end{array}$ & $\begin{array}{l}\text { Common duct obstruc- } \\
\text { tion-stone } \\
\text { Acute subsiding chole- } \\
\text { cystitis } \\
\text { Choledochostomy. Chole- } \\
\text { cystectomy }\end{array}$ \\
\hline 16 & 50 & M. & October & 31,1933 & 12.7 & 40 & 5.8 & + & $\begin{array}{l}\text { Chronic cholecystitis. } \\
\text { Postoperative biliary } \\
\text { fistula }\end{array}$ \\
\hline 17 & 49 & F. & December & 21,1932 & 20.6 & 35 & 4.5 & + & Common duct stone \\
\hline 18 & 59 & M. & $\begin{array}{l}\text { December } \\
\text { December } \\
\text { December } \\
\text { December }\end{array}$ & $\begin{array}{l}14,1933 \\
18,1933 \\
21,1933 \\
26,1933\end{array}$ & $\begin{array}{l}22.3 \\
21.0 \\
14.1 \\
14.6\end{array}$ & $\begin{array}{l}125 \\
150 \\
158 \\
\\
100\end{array}$ & $\begin{array}{l}15.7 \\
20.8 \\
21.1 \\
15.3\end{array}$ & $\begin{array}{l}+ \\
+ \\
+ \\
+\end{array}$ & $\begin{array}{l}\text { Postoperative stricture } \\
\text { Common duct } \\
\text { Operation December 18, } \\
1933\end{array}$ \\
\hline
\end{tabular}


TABLE III

The phosphatase content of the serum in hepatic jaundice

\begin{tabular}{|c|c|c|c|c|c|c|c|c|c|}
\hline 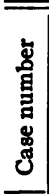 & \& & 8 & Dat & ate & 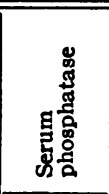 & 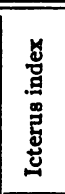 & 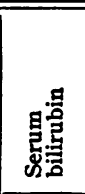 & 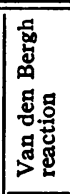 & Diagnosis \\
\hline & years & & & & $\begin{array}{l}\text { units per } \\
100 \text { cc. }\end{array}$ & & $\begin{array}{c}\text { mgm. } \\
\text { per. } \\
100 \text { cc. }\end{array}$ & & \\
\hline 19 & 27 & M. & $\begin{array}{l}\text { February } \\
\text { February } \\
\text { March } \\
\text { March } \\
\text { March } \\
\text { March }\end{array}$ & $\begin{array}{r}23,1934 \\
25,1934 \\
2,1934 \\
6,1934 \\
10,1934 \\
28,1934\end{array}$ & $\begin{array}{l}44.8 \\
72.2 \\
35.6 \\
52.2 \\
68.4 \\
53.9\end{array}$ & $\begin{array}{l}300 \\
375 \\
273 \\
204 \\
167 \\
136\end{array}$ & $\begin{array}{l}46.8 \\
47.3 \\
42.4 \\
25.0 \\
14.0 \\
12.2\end{array}$ & $\begin{array}{l}+ \\
+ \\
+ \\
+ \\
+ \\
+\end{array}$ & Acute hepatitis \\
\hline 20 & 26 & M. & $\begin{array}{l}\text { March } \\
\text { March } \\
\text { April }\end{array}$ & $\begin{array}{r}7,1933 \\
22,1933 \\
7,1933\end{array}$ & $\begin{array}{l}54.5 \\
14.5 \\
50.7\end{array}$ & $\begin{array}{r}166 \\
64 \\
38\end{array}$ & $\begin{array}{r}17.3 \\
7.8 \\
2.5\end{array}$ & $\begin{array}{l}+ \\
+ \\
+\end{array}$ & Acute hepatitis \\
\hline 21 & 34 & M. & $\begin{array}{l}\text { April } \\
\text { May } \\
\text { May }\end{array}$ & $\begin{array}{r}24,1933 \\
3,1933 \\
24,1933\end{array}$ & $\begin{array}{r}20.4 \\
8.2 \\
8.6\end{array}$ & $\begin{array}{l}55 \\
21 \\
13\end{array}$ & $\begin{array}{r}18.0 \\
3.7 \\
3.2\end{array}$ & $\begin{array}{l}+ \\
+ \\
+\end{array}$ & Acute hepatitis \\
\hline 22 & 38 & M. & $\begin{array}{l}\text { September } \\
\text { October } \\
\text { October } \\
\text { October }\end{array}$ & $\begin{array}{r}16,1933 \\
2,1933 \\
12,1933 \\
17,1933\end{array}$ & \begin{tabular}{r|}
10.4 \\
10.2 \\
11.5 \\
8.4
\end{tabular} & $\begin{array}{r}125 \\
125 \\
100 \\
75\end{array}$ & \begin{tabular}{r|}
11.7 \\
22.5 \\
13.1 \\
7.4
\end{tabular} & $\begin{array}{l}+ \\
+ \\
+ \\
+\end{array}$ & Subacute hepatitis \\
\hline 23 & 10 & M. & May & 4,1933 & 28.8 & 200 & 5.0 & + & Acute hepatitis \\
\hline 24 & 51 & M. & April & 25,1932 & 12.4 & & 10.7 & + & Acute hepatitis \\
\hline 25 & 27 & M. & December & 26,1933 & 10.6 & 38 & 3.1 & + & Acute hepatitis \\
\hline 26 & 29 & F. & April & 25,1932 & 9.5 & & 6.8 & + & Acute hepatitis \\
\hline 27 & 42 & M. & $\begin{array}{l}\text { March } \\
\text { March } \\
\text { April } \\
\text { April } \\
\text { April }\end{array}$ & $\begin{array}{r}27,1933 \\
31,1933 \\
7,1933 \\
19,1933 \\
27,1933\end{array}$ & $\begin{array}{l}18.4 \\
13.9 \\
12.1 \\
13.3 \\
11.7\end{array}$ & \begin{tabular}{|r|}
105 \\
150 \\
86 \\
47 \\
30
\end{tabular} & $\begin{array}{l}7.3 \\
6.8 \\
5.4 \\
3.4 \\
2.7\end{array}$ & $\begin{array}{l}+ \\
+ \\
+ \\
+ \\
+\end{array}$ & $\begin{array}{l}\text { Subacute hepatitis, syph- } \\
\text { ilitic }\end{array}$ \\
\hline 28 & 48 & M. & $\begin{array}{l}\text { April } \\
\text { May } \\
\text { May } \\
\text { May }\end{array}$ & $\begin{array}{l}19,1933 \\
12,1933 \\
18,1933 \\
24,1933\end{array}$ & $\begin{array}{l}20.6 \\
12.2 \\
12.4 \\
11.8\end{array}$ & \begin{tabular}{|r|}
176 \\
150 \\
100 \\
30
\end{tabular} & \begin{tabular}{r|}
17.4 \\
18.8 \\
10.7 \\
4.0
\end{tabular} & $\begin{array}{l}+ \\
+ \\
+ \\
+\end{array}$ & Acute hepatitis, syphilitic \\
\hline 29 & 63 & M. & May & 15,1933 & 10.4 & 37 & 5.0 & + & $\begin{array}{l}\text { Syphilis, post-arsphena- } \\
\text { mine jaundice }\end{array}$ \\
\hline 30 & 22 & F. & October & 12,1933 & 58.1 & 71 & 4.4 & + & Acute septic hepatitis \\
\hline
\end{tabular}


ever, there was no definite correlation between the changes in the phosphatase and the bilirubin content of the serum.

Eight cases of hepatic cirrhosis were studied (Table IV) and the phosphatase was elevated in five. Normal readings were obtained in two cases of congenital hemolytic jaundice.

TABLE IV

The phosphatase content of the serum in hepatic cirrhosis and hemolytic jaundice

\begin{tabular}{|c|c|c|c|c|c|c|c|c|c|}
\hline 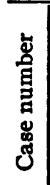 & 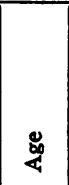 & $\mathscr{8}$ & $\mathrm{Da}$ & & 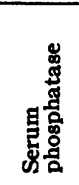 & 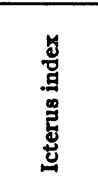 & 最曷 & 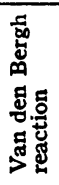 & Diagnosis \\
\hline & years & & & & $\begin{array}{l}\text { units } \\
\text { per } \\
100 \mathrm{cc} .\end{array}$ & & $\begin{array}{c}\underset{\text { per }}{\operatorname{mgm}} \\
100 \mathrm{cc} .\end{array}$ & & \\
\hline 31 & 37 & M. & $\begin{array}{l}\text { April } \\
\text { February } \\
\text { March } \\
\text { March } \\
\text { March } \\
\text { March }\end{array}$ & $\begin{array}{r}26,1933 \\
27,1934 \\
5,1934 \\
6,1934 \\
10,1934 \\
14,1934\end{array}$ & $\begin{array}{r}11.7 \\
12.4 \\
8.2 \\
13.5 \\
15.7 \\
13.9\end{array}$ & $\begin{array}{r}7 \\
136 \\
107 \\
115 \\
97 \\
136 \\
\text { (hem.) }\end{array}$ & $\begin{array}{r}1.4 \\
17.5 \\
14.6 \\
14.2 \\
7.9 \\
11.3\end{array}$ & $\begin{array}{l}0 \\
+ \\
+ \\
+ \\
+ \\
+\end{array}$ & $\begin{array}{l}\text { Portal cirrhosis. Ascites } \\
\text { Toxic hepatitis with } \\
\text { jaundice }\end{array}$ \\
\hline 32 & 33 & M. & February & 25,1933 & 1.3 & 122 & 30.5 & + & $\begin{array}{l}\text { Portal cirrhosis-No as- } \\
\text { cites. Toxic hepatitis } \\
\text { with jaundice }\end{array}$ \\
\hline 34 & 57 & M. & December & 22,1933 & 19.9 & 19 & 3.0 & 0 & $\begin{array}{l}\text { Portal cirrhosis of the } \\
\text { liver due to CCL poi- } \\
\text { soning. Ascites } \\
\text { Portal cirrhosis }\end{array}$ \\
\hline 35 & 40 & M. & July & 19,1933 & 11.8 & 17 & 3.5 & + & Portal cirrhosis. Ascites \\
\hline 36 & 59 & M. & October & 18,1933 & 15.4 & 11 & 2.0 & 0 & Portal cirrhosis. Ascites \\
\hline 37 & 29 & M. & January & 31,1933 & 8.6 & 15 & 2.0 & + & $\begin{array}{l}\text { Portal cirrhosis. No as- } \\
\text { cites. Wilson's disease }\end{array}$ \\
\hline 38 & 68 & M. & May & 16,1934 & 9.2 & 14 & 1.3 & \pm & $\begin{array}{l}\text { Obstructive biliary cir- } \\
\text { rhosis }\end{array}$ \\
\hline 39 & 14 & F. & April & 20,1934 & 9.9 & 167 & 24.4 & $\mathbf{0}$ & $\begin{array}{l}\text { Congenital hemolytic } \\
\text { jaundice }\end{array}$ \\
\hline 40 & 24 & M. & June & 13,1933 & 3.2 & 88 & 5.8 & 0 & $\begin{array}{l}\text { Congenital hemolytic } \\
\text { jaundice }\end{array}$ \\
\hline
\end{tabular}

The phosphatase was determined in bile obtained by duodenal drainage in a series of 15 cases of chronic cholecystitis without jaundice of one type or another (Table V). Phosphatase was present in all, but the amount varied widely, the lowest reading being 5 units per $100 \mathrm{cc}$. and the highest 
224. In those cases in which the duodenal and concentrated specimens were both studied the activity was increased in the latter. This would suggest that the phosphatase activity was increased with the concentration of bile in the gallbladder. The phosphatase activity was also determined in a few specimens of bile removed from the gallbladder at operation. These readings are not included in the table but were similar in magnitude to those reported there. Case 16 in Table V shows the phosphatase con-

TABLE V

The phosphatase content of bile obtained by duodenal intubation

\begin{tabular}{|c|c|c|c|c|c|c|c|}
\hline \multirow{2}{*}{$\begin{array}{l}\text { Case } \\
\text { num- } \\
\text { ber }\end{array}$} & \multirow[b]{2}{*}{ Age } & \multirow[b]{2}{*}{ Sex } & \multirow[b]{2}{*}{ Date } & \multicolumn{2}{|c|}{ Phosphatase } & \multicolumn{2}{|c|}{ Bile acids } \\
\hline & & & & $\begin{array}{c}\text { Duodenal } \\
\text { bile }\end{array}$ & $\begin{array}{c}\text { Concen- } \\
\text { trated } \\
\text { bile }\end{array}$ & $\begin{array}{c}\text { Duodenal } \\
\text { bile }\end{array}$ & $\begin{array}{c}\text { Concen- } \\
\text { trated } \\
\text { bile }\end{array}$ \\
\hline & years & & & $\begin{array}{l}\text { units per } \\
100 \text { cc. }\end{array}$ & $\begin{array}{l}\text { units per } \\
100 \mathrm{cc} .\end{array}$ & $\begin{array}{l}\text { mgm. per } \\
100 \mathrm{cc} .\end{array}$ & $\begin{array}{c}m g m . \text { per } \\
100 \text { cc. }\end{array}$ \\
\hline 1 & 27 & F. & November 22, 1933 & & 8 & & 465 \\
\hline 2 & 32 & F. & November 15,1932 & $\mathbf{0}$ & 5 & 180 & 484 \\
\hline 3 & 24 & F. & November 15,1932 & 44 & 224 & 240 & 265 \\
\hline 4 & 47 & F. & January 18,1933 & 5 & 102 & 74 & 182 \\
\hline 5 & 43 & F. & January & 51 & 61 & 99 & 93 \\
\hline 6 & 42 & F. & November 15,1933 & 25 & 97 & 80 & 91 \\
\hline 7 & 32 & F. & November 18,1933 & 5 & 199 & 82 & 488 \\
\hline 8 & 56 & F. & October $\quad 25,1933$ & 51 & 53 & $\mathbf{0}$ & 484 \\
\hline 9 & 32 & F. & October 25,1933 & 69 & 86 & 143 & 545 \\
\hline 10 & 24 & F. & December 6,1933 & 5 & 130 & $\mathbf{0}$ & 408 \\
\hline 11 & 55 & F. & November 29, 1933 & 13 & 85 & $\mathbf{0}$ & 164 \\
\hline 12 & 36 & F. & November 19,1933 & $\mathbf{0}$ & 5 & 23 & 438 \\
\hline 13 & 34 & F. & November 22, 1933 & 22 & 28 & 50 & 188 \\
\hline 14 & 62 & F. & November 22, 1933 & 28 & 30 & 59 & 96 \\
\hline 15 & 35 & M. & November 29,1933 & 16 & 29 & 83 & 182 \\
\hline 16 & 55 & F. & July $\quad 27,1933$ & 59 & & 335 & \\
\hline
\end{tabular}

tent of bile obtained by surgical drainage of the gallbladder in a patient with acute pancreatitis and total destruction of the pancreas.

\section{DISCUSSION}

The finding of phosphatase in bile obtained by duodenal drainage is not conclusive evidence for the hepatic origin of the enzyme. Bile obtained from the gallbladder at operation frequently contains amylase and it is usually assumed that the presence of amylase indicates the back flow of a small amount of pancreatic juice into the gallbladder. We have found phosphatase in bile obtained from the gallbladder at operation and this too may be extra-hepatic in origin. On the other hand the constant finding of phosphatase in specimens of bile, whether obtained at operation or by duodenal drainage, and the greater concentration in specimens from the gallbladder speaks for the hepatic origin of a considerable part of the en- 
zyme. Certainly the constancy of these observations would be difficult to explain on the assumption that the whole of the phosphatase came from pancreatic or duodenal juice which was present as a contaminant. The complete destruction of the pancreas in the case cited would further serve to exclude this organ as the source of the phosphatase found in the bile.

The present results confirm the earlier studies of Roberts (8) in demonstrating an increase in the phosphatase content of the serum in obstructive jaundice. This increase is further evidence for the hepatic origin of the phosphatase. The phosphatase content of the serum, however, contrary to the findings of Roberts (8) and in accord with those of Bodansky and Jaffe (2), is increased in cases of hepatitis as well as in obstructive jaundice. In our experience the test has been valueless in the differential diagnosis of the two types of jaundice. Hartman (5) has found that the serum phosphatase is increased in animals with experimentally produced cirrhosis of the liver. These observations are in agreement with our findings of an increase in the serum phosphatase in cases of hepatic cirrhosis without jaundice. These findings, together with the lack of correlation between the increase in the phosphatase and the elevation of the serum bilirubin, further suggest that in cirrhosis the serum phosphatase is a measure of hepatic disturbance rather than a consequence of the jaundice per se.

The finding of normal values in the two cases of congenital hemolytic icterus further emphasizes both the independence between the phosphatase and bilirubin and the difference in the pathogenesis of hemolytic icterus and of the obstructive and hepatic types of jaundice.

\section{SUM MARY}

Phosphatase was present in samples of bile obtained from the gallbladder at operation or by duodenal intubation.

The phosphatase content of the serum was increased in cases of jaundice due to hepatitis or to obstruction of the biliary passages. This test was of no value in the differential diagnosis of these two conditions. The phosphatase content of the serum was not increased in cases of hemolytic jaundice.

The phosphatase content of the serum was increased in cases of portal cirrhosis.

These findings suggest that the phosphatase in the bile probably is hepatic in origin and that some of the phosphatase normally present in serum is non-osseous and possibly hepatic in origin.

\section{BIBLIOGRAPHY}

1. Bodansky, A., Phosphatase studies. II. Determination of serum phosphatase. Factors influencing the accuracy of the determination. J. Biol. Chem., 1933, 101, 93. 
2. Bodansky, A., and Jaffe, H. L., Phosphatase studies. IV. Serum phosphatase of non-osseous origin. Significance of the variations of serum phosphatase in jaundice. Proc. Soc. Exper. Biol. and Med., 1933, 31, 107.

3. Bodansky, A., and Jaffe, H. L., Significance of clinical and experimental serum phosphatase variations; their osseous and non-osseous origins. J. Biol. Chem., Proc., 1934, 105, xi.

4. Demuth, P., Uber Phosphatstoffwechsel. I. Uber Hexosephosphatasen in menschlichen Organen und Körperflüssigkeiten. Biochem. Ztschr., 1925, $159,415$.

5. Hartman, F. W., Personal communication to the authors.

6. Kay, H. D., Phosphatase in growth and disease of bone. Physiol. Rev., 1932, 12, 384.

7. Roberts, W. M., Variations in the phosphatase activity of the blood in disease. Brit. J. Exper. Path., 1930, 11, 90.

8. Roberts, W. M., Blood phosphatase and the Van den Bergh reaction in the differentiation of the several types of jaundice. Brit. Med. J., 1933, 1, 734.

9. Robison, R., Possible significance of hexose phosphoric esters in ossification. Biochem. J., 1923, 17, 286. 